\section{Ligadura da artéria esfenopalatina via endoscópica no tratamento da epistaxe posterior severa}

\author{
Richard L. Voegels', Daniela C. Thoméz, \\ Patsy Priscilla V. Iturralde ${ }^{3}$, Ossamu Butugan ${ }^{4}$
}

\section{Endoscopic ligature of the sphenopalatine artery for severe posterior epistaxis}

Resumo / Summary

O bjetivo: Apresentar a experiência do Departamento de Otorrinolaringologia do HC-FMUSP na realização da ligadura da artéria esfenopalatina no tratamento da epistaxe posterior severa. Forma de estudo: Clínico prospectivo. Material e Método: Trinta e um pacientes submetidos a ligadura da artéria esfenopalatina via endoscópica, no período entre novembro de 1996 e julho de 2001, foram analisados de maneira restrospectiva. Resultados: Dezenove (61,3\%) pacientes eram do sexo masculino e $12(38,7 \%)$ do feminino, com idade variando de 15 a 80 anos (média de 44,7 anos). O procedimento cirúrgico foi realizado de maneira unilateral em 29 pacientes e bilateral em 2 pacientes, totalizando 33 ligaduras. A identificação da artéria foi possível em todos os casos, sendo alcançado o sucesso terapêutico com a técnica isolada. Conclusão: A ligadura da artéria esfenopalatina via endoscópica tem se mostrado uma técnica cirúrgica eficaz e segura no tratamento da epistaxe posterior severa.
Palavras-chave: epistaxe, ligadura da artéria esfenopalatina, tratamento.

Key words: epixtasis, endoscopic ligature of the sphenopalatine artery, treatment.

\footnotetext{
${ }^{1}$ Professor Doutor do Departamento de Otorrinolaringologia da Faculdade de Medicina da Universidade de São Paulo e Diretor de Rinologia do Departamento de Otorrinolaringologia do Hospital das Clínicas da Faculdade de Medicina da Universidade de São Paulo

${ }^{2}$ Doutoranda do Departamento de Otorrinolaringologia da Faculdade de Medicina da Universidade de São Paulo. ${ }^{3}$ Otorrinolaringologista formada pelo Departamento de Otorrinolaringologia da Faculdade de Medicina da Universidade de São Paulo.

${ }^{4}$ Professor Associado do Departamento de Otorrinolaringologia da Faculdade de Medicina da Universidade de São Paulo. Autor para correspondência: Richard Louis Voegels - Rua Iubatinga 145 ap 61-B São Paulo SP 05716-110 Fax: (0xx11) 3749-1029 - E-mail: voegels@attglobal.net

Artigo recebido em 01 de junho de 2001. Artigo aceito em 19 de setembro de 2002.
} 


\section{INTRODUÇÃO}

A epistaxe severa, em geral proveniente da região posterior da fossa nasal, consiste em uma das principais urgências otorrinolaringológicas e requer cuidados especiais ${ }^{1}$. O tratamento cirúrgico da epistaxe, o qual abrange a cauterização via microscópica ou endoscópica, ligadura arterial e embolização, normalmente fica reservado para pacientes que apresentam sangramento refratário a manobras conservadoras $^{1,2}$. No entanto, dependendo da severidade do quadro, a abordagem cirúrgica pode se tornar a primeira opção de tratamento.

A ligadura da artéria esfenopalatina através da microcirurgia foi introduzida por Prades ${ }^{3}$ em 1970. Sulsenti et al. ${ }^{4}$, em 1987, utilizaram o espéculo bivalvular de Prades e o microscópio para a ligadura da artéria esfenopalatina no meato médio, enquanto Budrovich e Saetti ${ }^{5}$, em 1992, foram os primeiros a relatar o uso do endoscópio para a realização deste procedimento. O objetivo da ligadura consiste na interrupção da irrigação nasal em um ponto distal suficiente para prevenir o fluxo direto, retrógrado e anastomótico dos sistemas carotídeos ipsi e contralaterais e representa uma excelente opção cirúrgica no manuseio da epistaxe posterior severa.

O presente estudo visa apresentar a experiência do Departamento de Otorrinolaringologia do HC-FMUSP na ligadura da artéria esfenopalatina via endoscópica endonasal no tratamento da epistaxe posterior severa.

\section{MATERIAL E MÉTODO}

Foi realizado um estudo retrospectivo nos pacientes submetidos a ligadura da artéria esfenopalatina via endoscópica endonasal no Departamento de Otorrinolaringologia do HC-FMUSP no período de novembro de 1996 a julho de 2001 ( 56 meses). A ligadura foi realizada em um total de 31 pacientes, 19 (61,3\%) do sexo masculino e 12 $(38,7 \%)$ do feminino, com idade variando de 15 a 80 anos (média de 44,7 anos). Todos os pacientes foram submetidos, previamente à cirurgia, a tratamento conservador sem sucesso (tampão anterior e/ou tampão ântero-posterior). Através da revisão dos prontuários foram obtidas informações sobre possíveis fatores predisponentes.

Todas as cirurgias foram realizadas sob anestesia geral, sem nenhum cuidado específico com a pressão arterial (paciente mantido normotenso). A vasocontricção tópica foi feita com algodões embebidas em adrenalina 1:2000, não sendo utilizado infiltrações. O princípio básico da técnica cirúrgica, que envolve a utilização do endoscópio nasal rígido de zero grau e $4 \mathrm{~mm}$ de diâmetro, consiste na identificação dos ramos da artéria esfenopalatina (Figura 1). Realiza-se incisão vertical inferior à porção posterior do corneto médio, $1 \mathrm{~cm}$ anterior à região mais caudal (Figura 2A). Elevando-se um retalho mucoperiosteal posterior e superiormente, o feixe fibroneurovascular é alcançado junto ao foramen esfenopalatino (Figura 2B). Após isolar a artéria e seus ramos, "clips" vasculares (Titanium Clip, Ethicon Endo-Surgery; INC; REF:LT200) são colocados sob visão direta (Figura 2C), e o retalho mucoperiosteal retorna a sua posição original (Figura 2D).

\section{RESULTADOS}

Dezesseis (51,6\%) pacientes foram submetidos a tampão antero-posterior, realizado com sonda de foley posteriormente, e rayon vaselinado anteriormente, e todos os pacientes haviam sido previamente tratados com tampão anterior. Cauterização elétrica via endoscópica de pontos sangrantes foi realizada em 6 (19,3\%) pacientes. Transfusão sanguínea foi necessária em 6 (19,3\%) pacientes.

A ligadura da artéria esfenopalatina via endoscópica endonasal foi realizada de forma unilateral em 29 (93,5\%) pacientes e bilateral em $2(6,4 \%)$ casos, somando um total de 33 ligaduras. A identificação dos ramos da artéria esfenopalatina foi possível em todos os casos, sendo alcançado o sucesso terapêutico com a técnica isolada. Em nenhum paciente foi mantido tamponamento nasal no pósoperatório. Não houve complicações durante ou após a cirurgia relacionada ao procedimento. Os pacientes receberam alta após 48hs ou mais de observação.

\section{DISCUSSÃO}

Cada vez mais, um maior número de otorrinolaringologistas considera a ligadura vascular precoce uma alternativa para determinados pacientes com epistaxe posterior se-

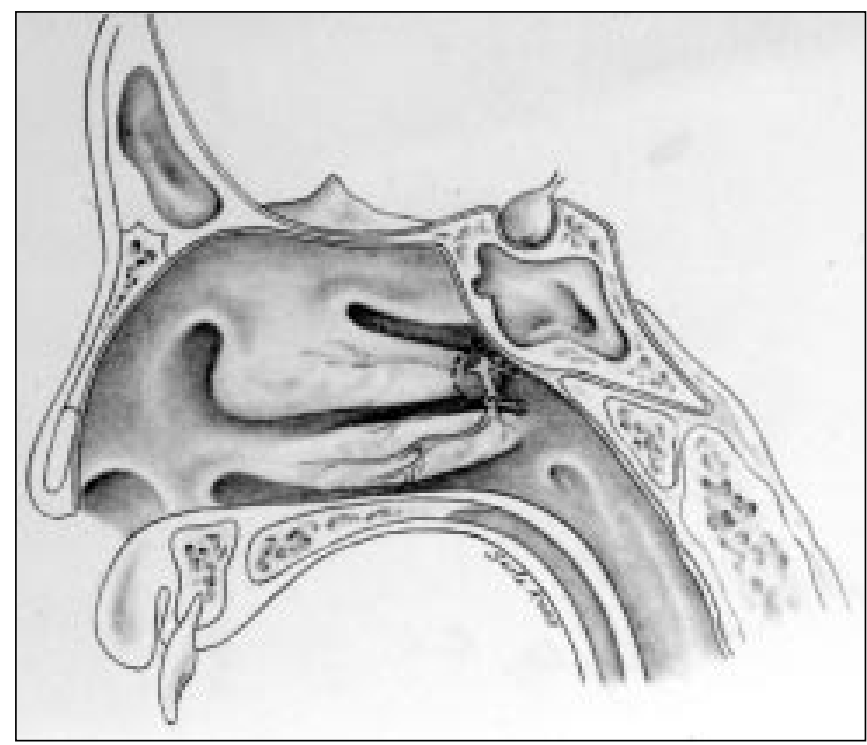

Figura 1. Vista da parede lateral da fossa nasal que mostra a relação do foramen esfenopalatino com o corneto médio, e os ramos da artéria esfenopalatina. 
Tabela 1. Características e fatores predisponentes dos pacientes estudados.

\begin{tabular}{|c|c|c|c|c|c|c|c|}
\hline Pacientes & Idade(anos) & Sexo & Hipertensão & $\begin{array}{l}\text { Diabetes } \\
\text { Mellítus }\end{array}$ & $\begin{array}{c}\text { Distúrbios } \\
\text { hematológicos }\end{array}$ & $\begin{array}{l}\text { Pós-operatório de } \\
\text { Turbinectomia } \\
\text { Inferior }\end{array}$ & Trauma \\
\hline 1 & 58 & $\mathrm{~F}$ & Sim & Não & Não & Sim & Não \\
\hline 3 & 64 & M & Sim & Sim & Não & Não & Não \\
\hline 4 & 20 & $\mathrm{~F}$ & Não & Não & $\begin{array}{c}\text { Doença de } \\
\text { Von-Willebrand }\end{array}$ & Não & $\operatorname{Sim}$ \\
\hline 7 & 32 & M & Não & Não & Não & Sim & Não \\
\hline 8 & 56 & M & Sim & Não & Não & Não & Sim \\
\hline 9 & 36 & M & Não & Não & Não & Sim & Não \\
\hline 10 & 22 & $\mathrm{~F}$ & Não & Não & Não & Não & Não \\
\hline 11 & 38 & M & Não & Não & Não & Sim & Não \\
\hline 12 & & M & Sim & Não & Não & Não & Não \\
\hline 18 & 20 & $\mathrm{~F}$ & Não & Não & Não & Sim & Não \\
\hline 19 & 42 & M & Não & Não & Não & Não & Sim \\
\hline 20 & 52 & $\mathrm{~F}$ & Sim & Não & Não & Não & Não \\
\hline 21 & 49 & $M$ & Sim & Não & Não & Não & Não \\
\hline 22 & 41 & $M$ & Não & Não & Não & Não & Sim \\
\hline 23 & 28 & $\mathrm{~F}$ & Não & Não & Não & Sim & Não \\
\hline 24 & 15 & $M$ & Não & Não & Não & Sim & Não \\
\hline 25 & 71 & $\mathrm{~F}$ & Sim & Não & Não & Não & Não \\
\hline 26 & 63 & $\mathrm{~F}$ & Sim & Não & Não & Não & Não \\
\hline 27 & 27 & $M$ & Não & Não & Não & Sim & Não \\
\hline 28 & 58 & $M$ & Sim & Não & Não & Não & Não \\
\hline 29 & 31 & $M$ & Não & Não & Não & Sim & Não \\
\hline
\end{tabular}

F: feminino; M: masculino

vera. Este fato se deve à possibilidade de maior conforto para o paciente, diminuir a hospitalização e a necessidade de transfusão sanguínea, reduzir a morbidade e até mesmo a mortalidade. Algumas indicações são apontadas na ligadura vascular, de forma similar às indicadas para a cauterização endoscópica descrita por O'Leary-Stickney et al.'? a) discrasia sanguínea que contraindica o tamponamento ou cirurgia aberta; b) sangramento refratário com tampão posicionado; c) hemorragia após cirurgia nasal; e d) intolerância do paciente ao tamponamento.

O tratamento cirúrgico para a epistaxe severa também está associado a diversos fatores predisponentes. Em nosso estudo, hipertensão, distúrbios hematológicos e trauma estiveram presentes em $16(51,6 \%), 1(3,2 \%)$ e $4(12,9 \%)$ pacientes, respectivamente. Epistaxe conseqüente a turbinectomia inferior foi observada em $11(35,4 \%)$ pacientes. Além disto, Barlow et al. ${ }^{1}$ observaram que o tratamento cirúrgico para a epistaxe severa está fortemente associado a sangramento posterior e transfusão sanguínea. Neste estudo, todos os pacientes apresentaram epistaxe posterior, porém apenas 19,3\% necessitaram de transfusão sanguínea.

A ligadura da artéria esfenopalatina via endoscópica tem se mostrado uma técnica cirúrgica efetiva no tratamento da epistaxe posterior severa, como pode ser observado em nosso primeiro estudo9. Em 1992, Budrovich et al. ${ }^{5}$ descreveram algumas dificuldades em isolar a artéria, mas não observaram complicações. No entanto, com o aumento da experiência na cirurgia endoscópica e nos parâmetros anatômicos, nenhuma dificuldade foi encontrada em isolar a artéria em nosso estudo. Acreditamos que esta técnica cirúrgica é simples, promove uma boa exposição do campo cirúrgico, apresenta baixa morbidade e deve ser considerada precocemente no manuseio da epistaxe posterior severa. 
Tabela 2. Tratamentos realizados previamente a realização da ligadura da artéri esfenopalatina e necessidade de transfusão sanguínea.

\begin{tabular}{ccccc}
\hline Pacientes & $\begin{array}{c}\text { Tampão } \\
\text { anterior }\end{array}$ & $\begin{array}{c}\text { Tampão } \\
\text { antero- } \\
\text { posterior }\end{array}$ & $\begin{array}{c}\text { Cauterização } \\
\text { elétrica }\end{array}$ & $\begin{array}{c}\text { Transfusão } \\
\text { sanguínea }\end{array}$ \\
\hline 1 & Sim & Sim (E) & Sim & Sim \\
2 & Sim & Sim (D) & Sim & Sim \\
3 & Sim & Sim (bilateral) & Não & Não \\
4 & Sim & Sim (E) & Sim & Sim \\
5 & Sim & Sim (D) & Não & Sim \\
6 & Sim & Sim (E) & Não & Sim \\
7 & Sim & Sim (E) & Sim & Não \\
8 & Sim & Sim (D) & Não & Não \\
9 & Sim & Sim (D) & Sim & Não \\
10 & Sim & Não & Sim & Não \\
11 & Sim & Não & Não & Não \\
12 & Sim & Sim (E) & Não & Não \\
13 & Sim & Sim (D) & Não & Não \\
14 & Sim & Não & Não & Sim \\
15 & Sim & Sim (bilateral) & Não & Não \\
16 & Sim & Sim (E) & Não & Não \\
17 & Sim & Sim (D) & Não & Não \\
18 & Sim & Sim (E) & Não & Não \\
19 & & Sim (bilateral) & Não & Não \\
\hline
\end{tabular}

D: fossa nasal direita; $E$ : fossa nasal esquerda; bilateral: ambas as fossas nasais
Tabela 3. Ligadura da artéria esfenopalatina via endoscópica endonasal.

\begin{tabular}{cccc}
\hline Pacientes & $\begin{array}{c}\text { Fossa nasal } \\
\text { abordada }\end{array}$ & $\begin{array}{c}\text { Identificação } \\
\text { da artéria }\end{array}$ & $\begin{array}{c}\text { Epistaxe no } \\
\text { pós-operatório }\end{array}$ \\
\hline 1 & Esquerda & Sim & Não \\
2 & Direita & Sim & Não \\
3 & Esquerda & Sim & Não \\
a Direita & Sim & Não \\
4 & Esquerda & Sim & Não \\
5 & Direita & Sim & Não \\
6 & Esquerda & Sim & Não \\
7 & Esquerda & Sim & Não \\
8 & Direita & Sim & Não \\
9 & Direita & Sim & Não \\
10 & Esquerda & Sim & Não \\
11 & Direita & Sim & Não \\
12 & Esquerda & Sim & Não \\
13 & Direita & Sim & Não \\
14 & Direita & Sim & Não \\
15 & Direita & Sim & Não \\
16 & Esquerda & Sim & Não \\
17 & Direita & Sim & Não \\
18 & Esquerda & Sim & Não \\
19 & Esquerda & & \\
& a Direita & & \\
\hline
\end{tabular}

D: fossa nasal direita; E: fossa nasal esquerda; bilateral: ambas as fossas nasals

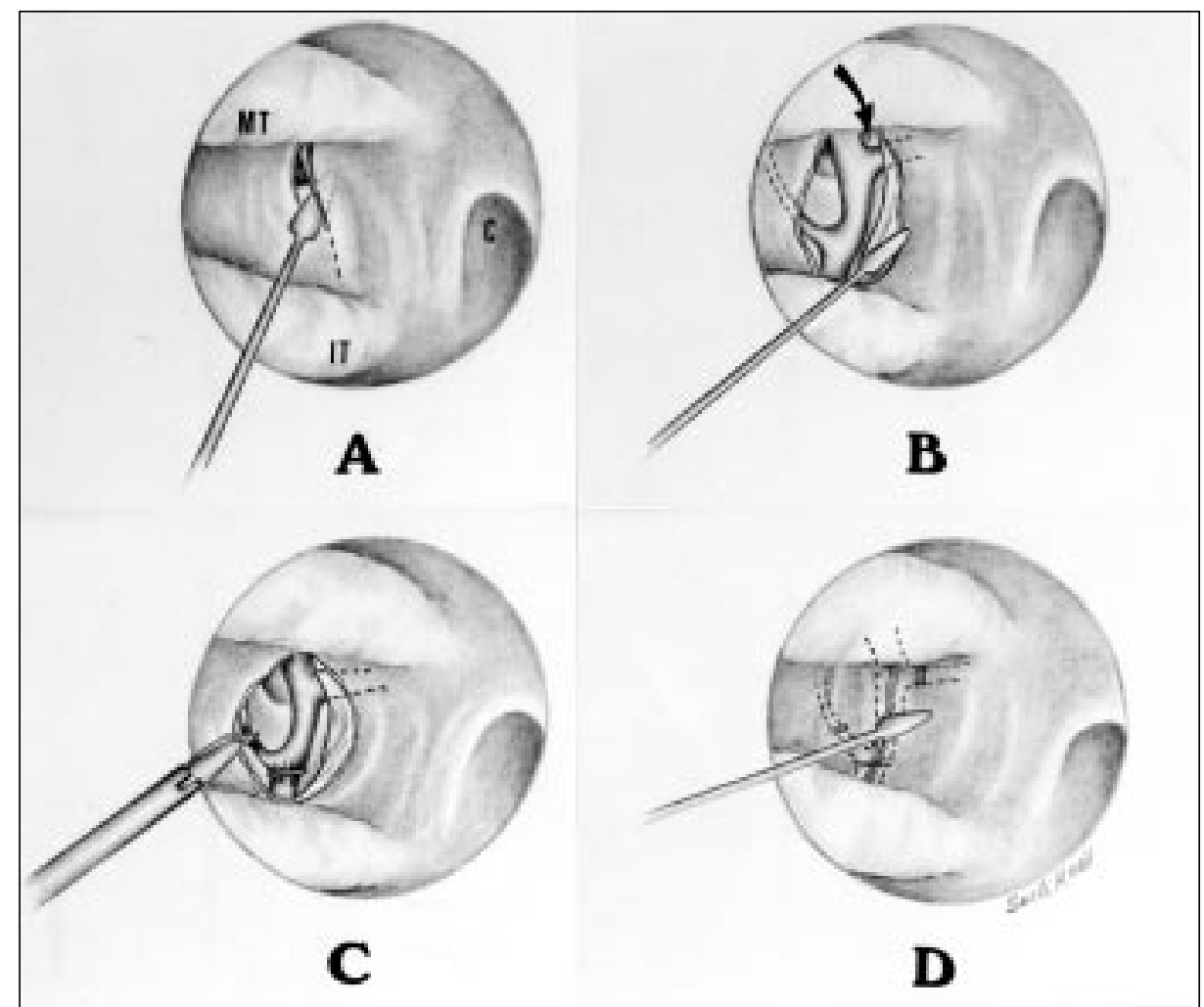

Figura 2. A. incisão vertical inferior à porção posterior do corneto médio; B. elevação de um retalho mucoperiosteal posterior e superiormente, sendo alcançada a artéria esfenopalatina em nível do foramen esfenopalatino; $\mathbf{C}$. aplicação de clips vasculares sob visão direta; $\mathbf{D}$. retorno do retalho mucoperiosteal à posição original (MT: corneto médio; IT: corneto inferior; C: coana; seta: indica o formen esfenopalatino) 


\section{REFERÊNCIAS BIBLIOGRÁFICAS}

1. Barlow DW, Deleyiannis FWB, Pinczower EF. Effectiveness of surgical management of epistaxis at a tertiary care center. Laryngoscope 107;21-24, 1997.

2. Sharp HR, Rowe-Jones JM, Biring GS et al. Endoscopic ligation or diametry of the sphenopalatine artery in persistent epistaxis. J Laryngol Otol 111:1047-1050, 1997.

3. Prades J. Abord endonasal de la fosse pterygo-maxillaire. LXXIII Cong Franc Compt Rendus des Seanc 290-296, 1976.

4. Sulsenyi G, Yanez C, Kadiri M. Recurrent epistaxis: microscopic endonasal clipping of the sphenopalatine artery. Rhinology 25:141-142, 1987.
5. Budrovich M, Saetti R. Microscopic and endoscopic ligature of the sphenopalatine artery. Laryngoscope 1390-1394, 1992.

6. Simpson GT, Janfaza P, Becker GD. Transantral sphenopalatine artery ligation. Laryngoscope 1001-1005, 1982.

7. O'Leary-Stickney K, Makielsky K, Weymuller EA. Rigid endoscopy for the control of epistaxis. Arch Otolaryngol Head Neck Surg 118:966-967, 1992

8. White PS. Endoscopic ligation of the sphenopalatine artery (ELSA): a preliminary description. J Laryngol Otol 110:27-30, 1996.

9. Voegels RV, Thomé DC, Iturralde PPV, Butugan O. Endoscopic ligature of the sphenopalatine artery for severe posterior epistaxis. Otolaryngol Head Neck Surg 124 (4):464-467, 2001. 